\title{
Patient characteristics of untreated chronic central serous chorioretinopathy patients with focal versus diffuse leakage
}

\author{
Thomas J. van Rijssen ${ }^{1}$ • Elon H. C. van Dijk ${ }^{1}$ Paula Scholz ${ }^{2}$ - Myrte B. Breukink ${ }^{3}$ • Rocio Blanco-Garavito ${ }^{4}$. \\ Eric H. Souied ${ }^{4}$. Robert E. MacLaren ${ }^{5}$. Giuseppe Querques ${ }^{4,6}$. Sascha Fauser ${ }^{2,7}$. Carel B. Hoyng ${ }^{3}$. \\ Susan M. Downes ${ }^{5}$. Camiel J. F. Boon ${ }^{1,8}$
}

Received: 5 February 2019 /Revised: 12 April 2019 / Accepted: 18 April 2019 / Published online: 23 May 2019

(C) The Author(s) 2019

\begin{abstract}
Purpose To describe the characteristics and potential differences between focal and diffuse phenotypes of untreated chronic central serous chorioretinopathy (cCSC).

Methods For this study, patients were divided in two groups. Focal leakage was defined as 1 "hot spot" of leakage, whereas diffuse leakage was defined as either $>1$ hot spot or a larger area of widespread leakage on FA. Clinical characteristics were assessed at presentation. After Bonferroni correction, $P$ values $<0.00125$ were deemed statistically significant.

Results The focal leakage group included 68 eyes (53 males), and the diffuse leakage group included 105 eyes ( 88 males). Mean best-corrected visual acuity (BCVA) was 77.1 \pm 8.1 Early Treatment of Diabetic Retinopathy Study (ETDRS) letters in the focal group and 76.0 \pm 9.6 ETDRS letters in the diffuse group $(p=0.440)$. In the focal group, mean age was $46.9 \pm 8.8$ years, whereas this was $49.7 \pm 8.3$ years in the diffuse group $(p=0.033)$. Mean central foveal thickness was $107.1 \pm 21.3 \mu \mathrm{m}$ in the focal group and $106.2 \pm 27.3 \mu \mathrm{m}$ in the diffuse group $(p=0.818)$. Mean choroidal thickness was $407.5 \pm 114.8 \mu \mathrm{m}$ in the focal group and $419.1 \pm 113.9 \mu \mathrm{m}$ in the diffuse group $(p=0.578)$. In the focal group, subretinal fluid was present in the fellow eye in $16 \%$ of the patients, as compared to $29 \%$ in the diffuse group $(p=0.067)$.

Conclusions In untreated cCSC patients with focal or diffuse leakage on FA, no marked differences in clinical characteristics were found. Extensive choroidal abnormalities may be present in both groups, which are presumed to lie at the basis of the development of cCSC.
\end{abstract}

Keywords Central serous chorioretinopathy $\cdot$ Diffuse leakage $\cdot$ Focal leakage $\cdot$ Fluorescein angiography $\cdot$ Indocyanine green angiography

\section{Introduction}

Central serous chorioretinopathy (CSC) is a chorioretinal disease characterized by an accumulation of subretinal fluid (SRF)

Camiel J. F. Boon

c.j.f.boon@lumc.nl

1 Department of Ophthalmology, Leiden University Medical Center, P.O. Box 9600, 2300 RC Leiden, The Netherlands

2 Department of Ophthalmology, University Hospital of Cologne, Cologne, Germany

3 Department of Ophthalmology, Radboud University Medical Center, Nijmegen, The Netherlands

4 Department of Ophthalmology, Centre Hospitalier Intercommunal de Creteil University Paris Est Creteil, Paris, France that often affects the macula [1-3]. Multimodal imaging techniques can provide a detailed assessment of the extent of retinal and choroidal abnormalities. In CSC, optical coherence tomography (OCT) is used to identify the presence of SRF, the

5 Oxford Eye Hospital, John Radcliffe Hospital, West Wing, Oxford, UK

6 Department of Ophthalmology, IRCCS Ospedale San Raffaele, Vita-Salute University, Milan, Italy

F. Hoffmann-La Roche, Basel, Switzerland

8 Amsterdam University Medical Center, Department of Ophthalmology, University of Amsterdam, Meibergdreef 9, Amsterdam, The Netherlands 
integrity of the anatomical layers of the retina, and possible changes in these layers such as outer retinal atrophy and/or posterior cystoid retinal degeneration [4]. Parameters on SDOCT may be helpful in predicting disease course in CSC [5].

SRF accumulation in CSC is the result of fluid leakage through a disrupted outer blood-retina barrier of the retinal pigment epithelium (RPE), while the underlying choroidal dysfunction seems to be a pivotal and probably primary abnormality in the pathogenesis of CSC. Therefore, both fluorescein angiography (FA) and indocyanine green angiography (ICGA) are valuable imaging techniques in the diagnosis, assessment of the disease activity and extent, as well as informing treatment choices in CSC [6-9]. FA can be used to detect the location, size, and number of "hot spots" of leakage and the extent of diffuse atrophic RPE alterations (DARA). ICGA can be performed to evaluate the underlying choroidal abnormalities $[8,10,11]$. Both FA and ICGA are therefore used to identify the area(s) for targeted treatment, for instance with photodynamic therapy (PDT) and high-density subthreshold micropulse laser (HSML) treatment $[7,12,13]$. In chronic CSC (cCSC), little is known about the clinical characteristics of patients with different phenotypes such as focal leakage or diffuse leakage on FA. If differences in the clinical characteristics and outcome of cCSC patients with focal leakage or diffuse leakage can be detected, this may also have prognostic and therapeutic consequences, and may give clues about potential difference in etiologies between these groups. The aim of this study is to describe the variety in abnormalities on FA and ICGA in the affected and fellow eye, to assess whether FA and ICGA abnormalities are comparable, and to compare clinical characteristics when dividing patients in groups based on focal or diffuse leakage on FA.

\section{Materials and methods}

Data from the "half-dose Photodynamic therapy versus highdensity subthreshold micropulse LAser treatment in patients with chronic CEntral serous chorioretinopathy" (PLACE) trial (ClinicalTrials.gov identifier: NCT01797861) was analyzed $[13,14]$. The PLACE trial was conducted from 2013 to 2016 at 5 academic medical centers, located in Leiden (the Netherlands), Nijmegen (the Netherlands), Cologne (Germany), Oxford (United Kingdom), and Paris (France). The study was performed in accordance with the tenets of the Declaration of Helsinki, and all participating centers received approval from their respective institutional review board and ethics committee. Written informed consent was obtained from all patients.

Patients who received previous CSC treatments (including oral medication such as eplerenone or spironolactone) or treatment with steroids within 3 months of screening were excluded. Additional inclusion and exclusion criteria are summarized in Table 1.

Patient demographics, BCVA in Early Treatment of Diabetic Retinopathy Study (ETDRS) letters, enhanced depth imaging (EDI) OCT, fundus autofluorescence (FAF), FA, and ICGA (Heidelberg Engineering, Heidelberg, Germany) were obtained at baseline. Eligible patients were divided in two groups, based on the leakage pattern on FA at baseline [15]. The group of patients with focal leakage had a maximum of 1 hot spot of leakage on FA. A hot spot of leakage was defined as an increase in the area of hyperfluorescence between early- and late-phase FA. Patients with diffuse leakage had either $>1$ hot spot of leakage or a larger area of hyperfluorescent leakage that could not be linked with a single spot of leakage on the FA image at $3 \mathrm{~min}$ after the start of infusion of fluorescein. DARA was defined as hyperfluorescent abnormalities on mid-phase FA [16]. The extent of DARA was divided in three groups based on their cumulative surface: first, $<1$ optic disc diameter; second, between 1 and 5 optic disc diameters; and the third group with $>5$ optic disc diameters of DARA. The degree of fluorescence on mid-phase ICGA (at $10 \mathrm{~min}$ after the start of infusion) at the location corresponding with the hot spot on FA was categorized in intense hyperfluorescence, intermediate hyperfluorescence, or absence of hyperfluorescence [17]. Additionally, the size of the abnormalities on ICGA $(<1$ optic disc diameter, $1-5$ optic disc diameters, and $>5$ optic disc diameters) was obtained. Patients were also categorized based on the correspondence of abnormalities on FA with findings on ICGA. The outcome of FAF imaging was categorized as either predominantly hyperautofluorescent, predominantly hypoautofluorescent, or a combination of hypo- and hyperautofluorescence, based on the degree of autofluorescence that was most common within the vascular arcades. Central foveal thickness (CFT) was defined as the distance between the inner borders of the internal limiting membrane and the inner border of the ellipsoid zone on SDOCT [18]. Subfoveal choroidal thickness (SFCT) was defined as the distance between the outer border of the RPE layer and the inner border of the choroid-sclera junction on enhanced depth imaging SD-OCT. Both CFT and SFCT were measured with the build-in caliper in the Heidelberg OCT machine software.

For the first 30 patients, FA and ICGA imaging were independently assessed by two experienced observers (EHCvD and TJvR). When there was no consensus, a third experienced observer (CJFB) had the decisive vote. An interobserver agreement calculation was performed for these first 30 patients. Thereafter, all imaging was assessed by TJvR and discussed with the senior author (CJFB) if deemed necessary. When ambiguous, the "draw region" tool in Heidelberg was used to determine the size of the optic disc and the size of abnormalities on FA and ICGA. 
Table 1 Inclusion and exclusion criteria of chronic central serous chorioretinopathy patients that were enrolled to this study

\begin{tabular}{ll}
\hline Inclusion criteria & Exclusion criteria \\
\hline Male or female cCSC patients $\geq 18$ years old & Previous treatments for CSC \\
Visual loss and/or presence of SRF on OCT $>6$ weeks & Intraretinal edema \\
Foveal SRF & Myopia $<-6$ diopters \\
At least one hyperfluorescent area of leakage on FA & Evidence of another diagnosis that \\
with RPE window defects typical for cCSC & could explain the vision loss or SRF \\
Best-corrected visual acuity (BCVA) of $>20 / 200$ in & Continuous or progressive visual loss \\
Snellen equivalent & or presence of SRF $>18$ months \\
Hyperfluorescent areas on ICGA & Currently treated or treated with corticosteroids within \\
& the last 3 months prior to screening visit \\
Sufficient quality of FA and ICGA imaging & Presence of soft drusen or signs of neovascularization \\
& Contraindications to receive FA, ICGA or PDT \\
\hline
\end{tabular}

(c) CSC (chronic) central serous chorioretinopathy, FA fluorescein angiography, ICGA indocyanine green angiography, $P D T$ photodynamic therapy, $S R F$ subretinal fluid

\section{Statistical analyses}

The statistical analyses were performed with SPSS Statistics (IBM Corp. version 23.0. Armonk, New York, United States of America). Independent $t$ test was used to compare continuous variables and Fisher exact tests for categorical variables between the focal leakage and diffuse leakage groups. After Bonferroni correction for 40 parameters, $P$ values lower than $0.00125(0.05 / 40)$ were considered to be statistically significant.

\section{Results}

In the PLACE trial, from November 2013 to September 2016, a total of 309 patients were screened for eligibility. After applying the inclusion and exclusion criteria, 141 male and 32 female patients could be included in this study. The 173 included patients had a mean age of $48.6 \pm 8.6$ years. The mean duration from the start of ocular complaints until baseline study visit was $7.1 \pm 4.4$ months. At baseline, mean BCVA was $76.4 \pm 9.0$ ETDRS letters, mean CFT was 106.6 \pm $25.1 \mu \mathrm{m}$, and mean SFCT was $414.7 \pm 113.9 \mu \mathrm{m}$.

\section{Interobserver agreement}

For the first 30 patients that were assessed, the interobserver agreement of focal versus diffuse disease on FA was $90 \%$. Cohen's kappa coefficient was used to assess the interobserver agreements for focal versus diffuse disease, which was found to be 0.80 . The first observer (TJvR) re-assessed the imaging of the first 30 patients with regard to focal or diffuse leakage at 6 months after initial assessment. In $97 \%$ of the patients, the categorization was identical to the results of the first assessment. For DARA size on FA (smaller than 1 optic disc diameter, between 1 and 5 optic disc diameters, or larger than 5 optic disc diameters), an intraclass correlation coefficient was calculated using a two-way mixed model. There was agreement between the two observers in $87 \%$ of patients, with an intraclass correlation coefficient of 0.80 .

\section{Clinical characteristics of the focal leakage and diffuse leakage groups}

Sixty-eight patients were categorized as having focal leakage (focal leakage group) and 105 patients were categorized as having diffuse leakage on FA (diffuse leakage group). There were 23 out of 68 patients (33.8\%) in the focal leakage group with a previous CSC episode, and 25 out of 104 patients (24\%) in the diffuse leakage group with a previous episode of CSC (in 1 patient the information was unavailable), which was not a statistically significant difference between the groups ( $p=0.162)$. Characteristics of the patient groups with focal leakage and diffuse leakage on FA are depicted in Fig. 1. In the patients with focal leakage on FA, a mean age of $46.8 \pm$ 8.8 years, a mean duration of symptoms until cCSC diagnosis of 7.3 \pm 4.7 months, a mean BCVA of $77.1 \pm 8.1$ ETDRS letters, a mean CFT of $107.1 \pm 21.3 \mu \mathrm{m}$, and a mean SFCT of $407.5 \pm 114.8 \mu \mathrm{m}$ were found. CFT could not be measured in 1 patient and SFCT could not be measured in 19 patients, due to suboptimal quality of EDI-OCT imaging. At the screening visit, patients with diffuse leakage on FA had a mean age of $49.7 \pm 8.2$ years, a mean duration of symptoms until cCSC diagnosis of $7.0 \pm 4.2$ months, a mean BCVA of $76.0 \pm 9.6$ ETDRS letters, a mean CFT of $106.2 \pm 27.3 \mu \mathrm{m}$, and a mean SFCT of $419.2 \pm 113.9 \mu \mathrm{m}$, none of which were significantly different from the focal leakage group after Bonferroni correction $(p=0.033, p=0.704, p=0.440, p=0.818, p=0.578$, respectively). Because of insufficient EDI-OCT imaging quality (the scleral border could not be determined), SFCT could not be obtained in 27 patients with diffuse leakage on FA. There were no significant differences $(p=0.423)$ between sex at 

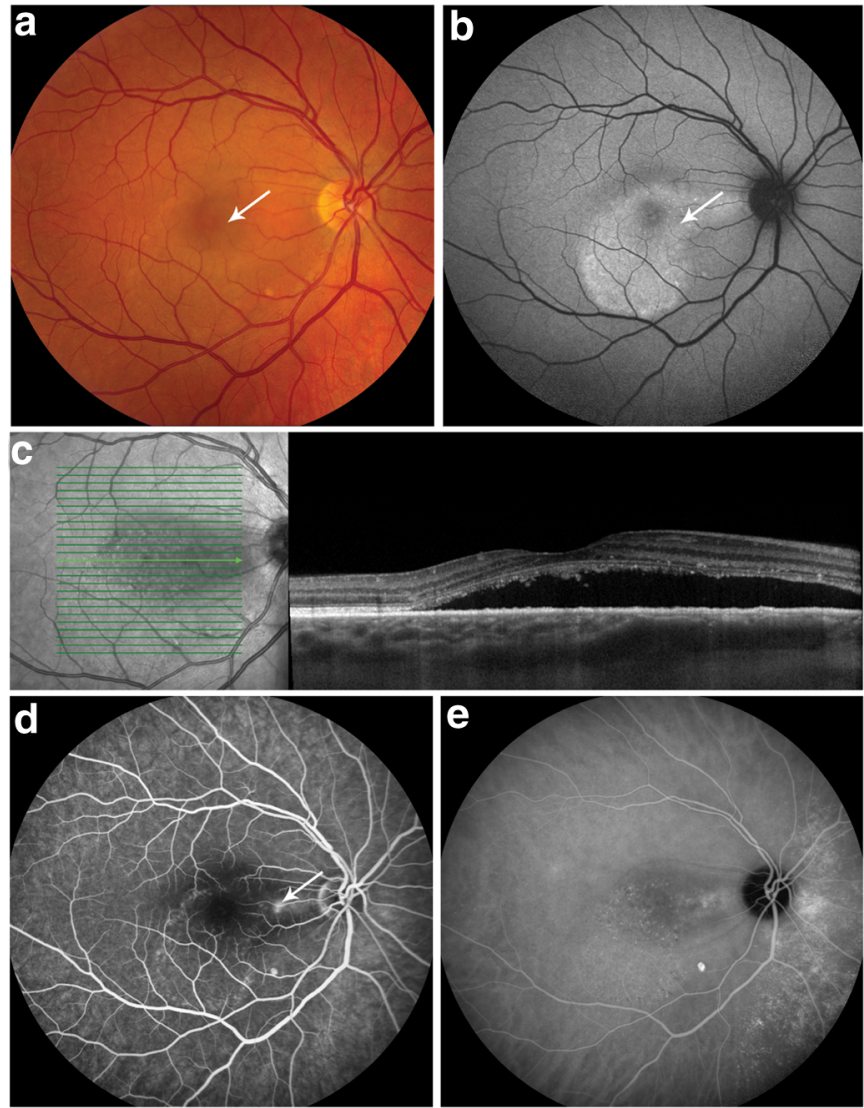

Fig. 1 Multimodal imaging of two chronic central serous chorioretinopathy (cCSC) patients who were considered to show focal leakage (a-e) and diffuse leakage ( $\mathbf{f}-\mathbf{j})$ on fluorescein angiography (FA). a-e A 51-year-old man with foveal retinal pigment epithelium (RPE) alterations on fundus photography (a) with a more extensive area of hyperautofluorescence on fundus autofluorescence (FAF), compared to the size of the pigment alterations (b). Foveal subretinal fluid with some hyperreflective subretinal debris was detected on optical coherence tomography (OCT; $\mathbf{c})$. Both FA (d) and indocyanine green angiography (ICGA; e) showed a focal leakage spot, indicated by the white arrow. In addition, hyperfluorescent retinal pigment epithelium alterations are

baseline between the focal leakage group (53 men, 77.9\%) and diffuse leakage group ( 88 men, $83.8 \%$ ). In the focal leakage group, there were 58 patients $(85.3 \%)$ with DARA $<1$ optic disc diameter, 9 patients (13.2\%) with DARA 1-5 optic disc diameters, and 1 patient (1.5\%) with DARA of $>5$ optic disc diameters on FA. In comparison, the diffuse leakage group consisted of 60 patients $(57.1 \%)$ with DARA $<1$ optic disc diameter, 35 patients (33.3\%) with DARA $1-5$ optic disc diameters, and 10 patients $(9.5 \%)$ with DARA of $>5$ optic disc diameters $(p<0.001, p=0.004$, and $p=0.052$, respectively, compared to the focal leakage group). In the focal leakage group, there were 56 patients $(82.4 \%)$ with foveal DARA, 12 patients $(17.6 \%)$ with DARA within 1 optic disc diameter from the fovea, and no patients with DARA outside the fovea, as compared to 91 patients $(86.7 \%), 12$ patients $(11.4 \%)$, and 2 patients $(1.9 \%)$ with these characteristics in the diffuse leakage group, respectively $(p=0.516, p=0.269$, and $p=0.519$,
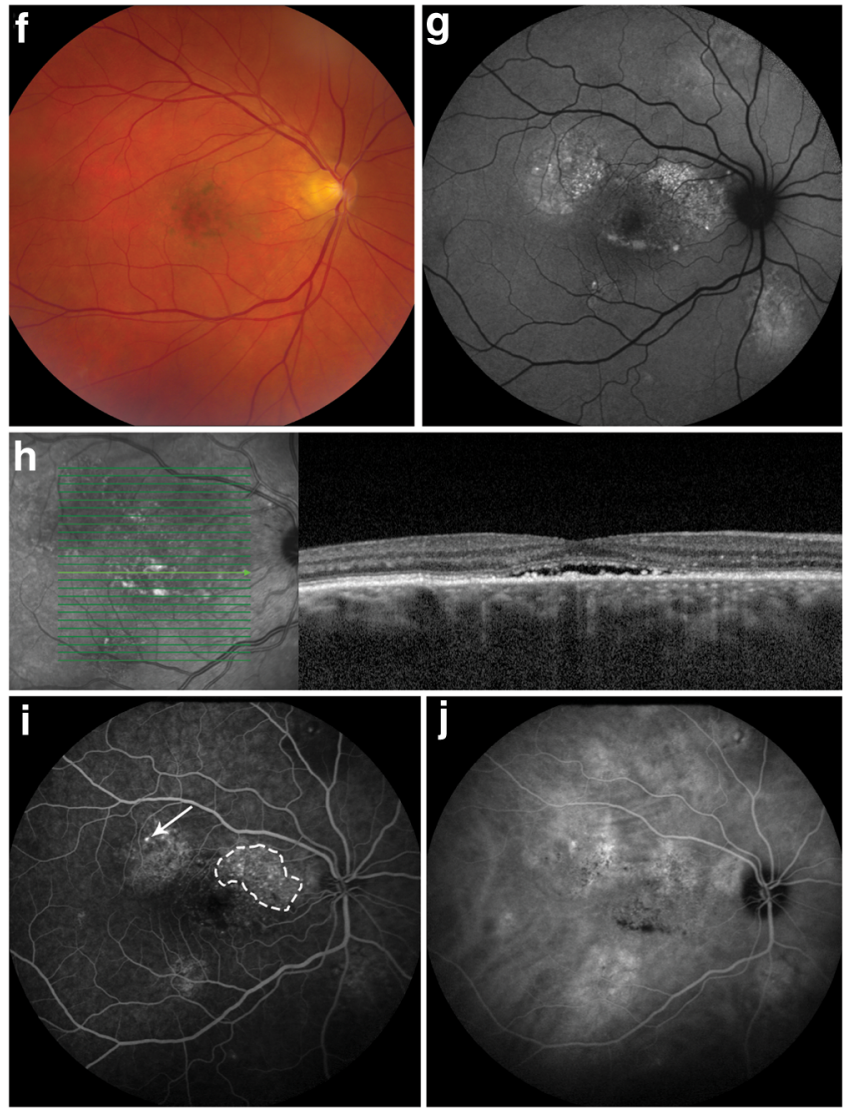

visible on FA (d). ICGA shows a more extensive area of hyperfluorescence compared to FA (e). f, g A 52-year-old man categorized as having diffuse leakage. RPE changes are visible on fundus photography (f). Both hyper- and hypoautofluorescent abnormalities are present on FAF (g). Foveal subretinal fluid is present on OCT, as well as some hyperreflective deposits and RPE irregularities (h). FA shows both a focal leakage spot (white arrow) and an area of diffuse hyperfluorescent leakage (within the dashed borders; i). Multiple areas of hyperfluorescence typical of cCSC are visible on ICGA (j), which are more extensive than the abnormalities on FA

respectively). In the focal leakage group, none of the patients had hypofluorescence on ICGA, while mild hyperfluorescence was observed in 6 patients $(8.8 \%)$, and intense hyperfluorescence was seen in 62 patients $(91.2 \%)$. In contrast, the diffuse leakage group consisted of 4 patients (3.8\%) with hypofluorescence on ICGA, 20 patients (19.2\%) with mild hyperfluorescence on ICGA $(p=0.081)$, and 81 patients $(77.1 \%)$ with intense hyperfluorescence on ICGA ( $p=0.023$, compared to the focal leakage group). The areas of hyperfluorescent abnormalities on ICGA were more extensive on FA in 30 of the patients $(44.1 \%)$ in the focal leakage group, whereas there were 58 patients $(55.2 \%)$ in the diffuse leakage group $(p=0.161)$. In all other patients, the size of the area of hyperfluorescent abnormalities on FA and ICGA was comparable, with none of the patients showing a smaller area of hyperfluorescent abnormalities on ICGA as compared to FA. The autofluorescence description on FAF was 
predominantly hypoautofluorescent in 27 patients (39.7\%), predominantly hyperautofluorescent in 17 patients $(25.0 \%)$, and both hyper- and hypoautofluorescent in 24 patients $(35.3 \%)$ in the focal leakage group, while this was the case in 28 patients (26.9\%), 29 patients (27.9\%), and 47 patients $(45.2 \%)$ in the diffuse leakage group, respectively. There were no significant differences between the degree of autofluorescence on FAF in patients with predominantly hypoautofluorescence $(p=0.095)$, predominantly hyperautofluorescence $(p=0.727)$, and both hyper- and hypoautofluorescence $(p=0.209)$ on FAF, between the focal and diffuse leakage groups.

\section{Characteristics of the fellow eye on multimodal imaging}

The characteristics of the fellow eye for both the focal leakage and diffuse leakage group are shown in Table 2. SRF in the fellow eye was present in 11 patients $(16.2 \%)$ in the focal leakage group, as compared to 30 patients $(29.1 \%)$ in the diffuse leakage group $(p=0.067)$. In the focal leakage group, there were 33 patients $(49.3 \%)$ without DARA in the fellow eye on FA, 24 patients $(35.8 \%)$ with DARA $<1$ optic disc diameter, 9 patients (13.4\%) with DARA between 1 and 5 optic disc diameters, and 1 patient $(1.5 \%)$ with DARA $>5$ optic disc diameters. In comparison, the diffuse leakage group consisted of 29 patients (28.2\%) without DARA in the fellow eye on FA $(p=0.009), 53$ patients $(51.5 \%)$ with DARA $<1$ optic disc diameters $(p=0.060), 14$ patients $(13.6 \%)$ with DARA between 1 and 5 optic disc diameters $(p>0.999)$, and 7 patients $(6.8 \%)$ with DARA $>5$ optic disc diameters $(p=0.151)$. There were no statistically significant differences between the focal and diffuse leakage group regarding the FAF characteristics (Table 2).

\section{Discussion}

In this study, cCSC patients were divided into two categories, based on early-phase FA abnormalities: focal leakage was defined as a maximum of one hot spot of leakage, whereas the diffuse leakage group comprised patients with either multiple hot spots of leakage or a larger area of leakage. We hypothesized that more severe and extensive disease would be present in the diffuse leakage group, with a potentially different prognosis and treatment response outcome. In our study, abnormalities on ICGA were never smaller than on FA in all patients in both groups. Most of the patients in both groups had multifocal hyperfluorescent abnormalities on ICGA outside the area of the hotspot on FA. No significant differences in BCVA, CFT, and SFCT between the focal and diffuse leakage group were found. The fellow eye of patients with diffuse leakage on FA was more often affected by SRF, although this difference did not reach the threshold of statistical significance.

In both the focal and the diffuse leakage group, none of the patients had a smaller area of abnormalities on ICGA compared to FA, which corresponds to findings in previous studies $[6,19]$. In addition, multifocal areas of hyperfluorescence on ICGA outside the zone of leakage on FA were often present in both groups. ICGA is primarily used to evaluate the status of the choroid, while FA can reveal both DARA and hot spots of fluorescein leakage $[10,20]$. For CSC, this suggests that choroidal abnormalities are generally more extensive than abnormalities of the RPE. Chronic CSC may arise as chronic choroidal thickening (pachychoroid) and dysfunction, such as hyperpermeability and congestion, gradually causing RPE damage. This pachychoroid and associated choroidal dysfunction may lead only to DARA without SRF leakage, which is then compatible with pachychoroid pigment epitheliopathy, which can be regarded as the forme fruste of CSC [21]. As a complication, the RPE outer blood-retina barrier can be damaged to such a degree that SRF leaks through an RPE defect, causing active SRF leakage in CSC. Since choroidal abnormalities on ICGA extend further than those on FA, guided treatment would underestimate the extent of the underlying disease and may lead to suboptimal treatment efficacy with a higher risk of recurrences due to inadequate treatment area coverage. ICGA-guided treatment may therefore be preferable for optimal treatment efficacy $[7,13]$. The observation that abnormalities on ICGA are generally at least as extensive as the RPE abnormalities on FA may also explain why PDT, which primarily targets the dysfunctional choroid, is more effective in achieving a complete resolution of SRF and leads to a better functional outcome as compared to HSML in the PLACE trial [13].

Patients with an absence of hyperfluorescent abnormalities on ICGA are less likely to respond favorably to PDT treatment $[22,23]$. Despite the fact that abnormalities on ICGA are often more widespread compared to FA, strongly suggesting that choroidal changes are underlying in $\mathrm{CSC}$, chronic damage to the RPE may also be of importance in the pathogenesis of SRF leakage even if choroidal hyperfluorescence and leakage become less pronounced in ICGA with time.

BCVA, CFT, and SFCT did not differ significantly between the focal and diffuse leakage groups, which may indicate that patients with diffuse leakage do not necessarily have a functionally more severe phenotype in comparison to patients with focal leakage [16]. The primary pathophysiological mechanism in CSC could thus be the same, resulting in dysfunction of the choroid and RPE, regardless of the CSC subtypes.

Despite the fact that this is the first phenotypic study on cCSC based on a large prospective multimodal imaging dataset, there are limitations to this study. The focal leakage and diffuse leakage categories that were designed for this study may be somewhat arbitrary. However, these categories 
Table 2 Demographics and characteristics of chronic central serous chorioretinopathy patients in the focal leakage and diffuse leakage group at presentation

\begin{tabular}{|c|c|c|c|c|}
\hline \multicolumn{2}{|l|}{ Affected eye } & $\begin{array}{l}\text { Focal leakage } \\
(n=68) \\
\text { Mean } \pm \text { SD }\end{array}$ & $\begin{array}{l}\text { Diffuse leakage } \\
(n=105) \\
\text { Mean } \pm \text { SD }\end{array}$ & $p$ value \\
\hline \multicolumn{2}{|l|}{ Age at screening (years) } & $46.9 \pm 8.8$ & $49.7 \pm 8.3$ & 0.033 \\
\hline \multicolumn{2}{|l|}{ Duration of symptoms until cCSC diagnosis (months) } & $7.3 \pm 4.7$ & $7.0 \pm 4.2$ & 0.704 \\
\hline \multicolumn{2}{|l|}{ BCVA in ETDRS letters } & $77.1 \pm 8.1$ & $76.0 \pm 9.6$ & 0.440 \\
\hline \multicolumn{2}{|l|}{ CFT $(\mu \mathrm{m})$} & $107.1 \pm 21.3$ & $106.2 \pm 27.3$ & 0.818 \\
\hline \multirow{2}{*}{\multicolumn{2}{|c|}{$\operatorname{SFCT}(\mu \mathrm{m})$}} & $407.5 \pm 114.8$ & $419.2 \pm 113.9$ & 0.578 \\
\hline & & Number $(\%)$ & Number $(\%)$ & \\
\hline Sex & Male & $\overline{53(77.9 \%)}$ & $\overline{88(83.8 \%)}$ & 0.423 \\
\hline \multirow[t]{3}{*}{ DARA on FA } & $<1$ optic disc diameter & $58(85.3 \%)$ & $60(57.1 \%)$ & $<0.001^{*}$ \\
\hline & $1-5$ optic disc diameter & $10(14.7 \%)$ & $45(42.9 \%)$ & 0.004 \\
\hline & $>5$ optic disc diameter & $1(1.5 \%)$ & $10(9.5 \%)$ & 0.052 \\
\hline \multirow[t]{3}{*}{ Location of DARA } & Fovea & $56(82.4 \%)$ & $90(86.5 \%)$ & 0.516 \\
\hline & $\begin{array}{l}\text { Within } 1 \text { optic disc diameter of the } \\
\text { fovea }\end{array}$ & $12(17.6 \%)$ & $12(11.5 \%)$ & 0.269 \\
\hline & Outside the fovea & $0(0.0 \%)$ & $2(1.9 \%)$ & 0.519 \\
\hline \multirow[t]{3}{*}{ Degree of hyperfluorescence on ICGA } & Hypofluorescence & $0(0.0 \%)$ & $4(3.8 \%)$ & 0.154 \\
\hline & Mild hyperfluorescence & $6(8.8 \%)$ & $20(19.2 \%)$ & 0.081 \\
\hline & Intense hyperfluorescence & $62(91.2 \%)$ & $80(76.9 \%)$ & 0.023 \\
\hline $\begin{array}{l}\text { Hyperfluorescent areas on ICGA outside of the leakage spot } \\
\text { on FA }\end{array}$ & Yes & $48(70.6 \%)$ & $91(87.5 \%)$ & 0.009 \\
\hline $\begin{array}{l}\text { Comparability of the size of the abnormalities on FA and } \\
\text { ICGA }\end{array}$ & Larger on ICGA & $30(44.1 \%)$ & $58(55.2 \%)$ & 0.161 \\
\hline \multirow[t]{3}{*}{ Degree of autofluorescence on FAF } & Predominantly hypoautofluorescent & $27(39.7 \%)$ & $28(26.9 \%)$ & 0.095 \\
\hline & Predominantly hyperautofluorescent & $17(25.0 \%)$ & $29(27.9 \%)$ & 0.727 \\
\hline & Both hyper- and hypoautofluorescent & $24(35.3 \%)$ & $47(45.2 \%)$ & 0.209 \\
\hline
\end{tabular}

$B C V A$ best-corrected visual acuity, $C F T$ central foveal thickness, $S F C T$ subfoveal choroidal thickness, DARA diffuse atrophic retinal pigment epithelium alterations, $F A$ fluorescein angiography, $F A F$ fundus autofluorescence, $I C G A$ indocyanine green angiography, $R P E$ retinal pigment epithelium, $S D$ standard deviation

$* P$ values $<0.00125$ were considered to be statistically significant

were based on the results of other studies, clinical experience, and observations of two independent graders [2, 10, 15, 24].

In conclusion, abnormalities on FA appear to be of similar size or larger on ICGA, in both focal and diffuse leakage groups, which may indicate primary dysfunction of the choroid. No significant differences in BCVA, CFT, and SFCT were found between cCSC with focal versus diffuse leakage, which may suggest that the type of leakage is not necessarily associated with a big difference in functional severity of disease. Our findings also indicate that these subgroups are likely to share the same pathophysiologic background. Further studies are needed to assess if there are differences in response to treatment and long-term outcome between these cCSC subgroups.

Role of funding organizations The funding organizations had no role in the design or conduct of the study; collection, management, analysis, and interpretation of the data; preparation, review, or approval of the manuscript; and decision to submit the manuscript for publication. They provided unrestricted grants.

Funding This research was supported by the following foundations: MaculaFonds, Retina Netherlands, BlindenPenning, and Landelijke Stichting voor Blinden en Slechtzienden, which contributed through
UitZicht, as well as Rotterdamse Stichting Blindenbelangen, Haagse Stichting Blindenhulp, ZonMw VENI Grant, and Gisela Thier Fellowship of Leiden University (CJFB).

For the PLACE trial, funding was received from Novartis Pharma B.V. (Arnhem, the Netherlands) solely for the purchase of verteporfin (Visudyne) to enable photodynamic therapy treatment at the Oxford site, because photodynamic therapy currently is not reimbursed routinely by the United Kingdom National Health Service for treating central serous chorioretinopathy. Novartis Pharma B.V. had no role in funding, designing, conducting, or evaluating the study, nor in the writing of this manuscript.

\section{Compliance with ethical standards}

Competing interests The authors declare that they have no conflict of interest.

Ethical approval All procedures performed in studies involving human participants were in accordance with the ethical standards of the institutional and/or national research committee and with the 1964 Helsinki declaration and its later amendments or comparable ethical standards. This study was approved by the institutional review boards and ethical committees of each participating center.

Informed consent Written informed consent was obtained from all individual participants included in the study. All patients were provided a means to opt out. 
Open Access This article is distributed under the terms of the Creative Commons Attribution 4.0 International License (http:// creativecommons.org/licenses/by/4.0/), which permits unrestricted use, distribution, and reproduction in any medium, provided you give appropriate credit to the original author(s) and the source, provide a link to the Creative Commons license, and indicate if changes were made.

\section{References}

1. Prunte C, Flammer J (1996) Choroidal capillary and venous congestion in central serous chorioretinopathy. Am J Ophthalmol 121(1):26-34

2. Nicholson B, Noble J, Forooghian F, Meyerle C (2013) Central serous chorioretinopathy: update on pathophysiology and treatment. Surv Ophthalmol 58(2):103-126. https://doi.org/10.1016/j. survophthal.2012.07.004

3. Imamura Y, Fujiwara T, Margolis R, Spaide RF (2009) Enhanced depth imaging optical coherence tomography of the choroid in central serous chorioretinopathy. Retina 29(10):1469-1473. https://doi. org/10.1097/IAE.0b013e3181be0a83

4. Spaide RF, Curcio CA (2011) Anatomical correlates to the bands seen in the outer retina by optical coherence tomography: literature review and model. Retina 31(8):1609-1619. https://doi.org/10. 1097/IAE.0b013e3182247535

5. Lee H, Lee J, Chung H, Kim HC (2016) Baseline spectral domain optical coherence tomographic hyperreflective foci as a predictor of visual outcome and recurrence for central serous chorioretinopathy. Retina 36(7):1372-1380. https://doi.org/10.1097/iae. 0000000000000929

6. Spaide RF, Hall L, Haas A, Campeas L, Yannuzzi LA, Fisher YL, Guyer DR, Slakter JS, Sorenson JA, Orlock DA (1996) Indocyanine green videoangiography of older patients with central serous chorioretinopathy. Retina 16(3):203-213

7. Yannuzzi LA, Slakter JS, Gross NE, Spaide RF, Costa D, Huang SJ, Klancnik JM Jr, Aizman A (2003) Indocyanine green angiographyguided photodynamic therapy for treatment of chronic central serous chorioretinopathy: a pilot study. Retina 23(3):288-298

8. Guyer DR, Yannuzzi LA, Slakter JS, Sorenson JA, Ho A, Orlock D (1994) Digital indocyanine green videoangiography of central serous chorioretinopathy. Arch Ophthalmol 112(8):1057-1062

9. Hua R, Yao K, Xia F, Li J, Guo L, Yang G, Tao J (2016) The hyperfluorescent transitional bands in ultra-late phase of indocyanine green angiography in chronic central serous chorioretinopathy. Lasers Surg Med 48(3):260-263. https://doi.org/10.1002/lsm.22434

10. Cardillo Piccolino F, Borgia L, Zinicola E, Zingirian M (1995) Indocyanine green angiographic findings in central serous chorioretinopathy. Eye (Lond) 9(Pt 3):324-332. https://doi.org/10. 1038/eye.1995.63

11. Gajdzik-Gajdecka U, Dorecka M, Nita E, Michalska A, MiniewiczKurowska J, Romaniuk W (2012) Indocyanine green angiography in chronic central serous chorioretinopathy. Med Sci Monit 18(2): Cr51-Cr57

12. Breukink MB, Mohr JK, Ossewaarde-van Norel A, den Hollander AI, Keunen JE, Hoyng CB, Boon CJ (2016) Half-dose photodynamic therapy followed by diode micropulse laser therapy as treatment for chronic central serous chorioretinopathy: evaluation of a prospective treatment protocol. Acta Ophthalmol 94(2):187-197. https://doi.org/10.1111/aos.12938

13. van Dijk EHC, Fauser S, Breukink MB, Blanco-Garavito R, Groenewoud JMM, Keunen JEE, Peters PJH, Dijkman G, Souied EH, MacLaren RE, Querques G, Downes SM, Hoyng CB, Boon
CJF (2018) Half-dose photodynamic therapy versus high-density subthreshold micropulse laser treatment in patients with chronic central serous chorioretinopathy: the PLACE trial. Ophthalmology 125(10):1547-1555. https://doi.org/10.1016/j. ophtha.2018.04.021

14. Breukink MB, Downes SM, Querques G, van Dijk EHC, den Hollander AI, Blanco-Garavito R, Keunen JEE, Souied EH, MacLaren RE, Hoyng CB, Fauser S, Boon CJF (2015) Comparing half-dose photodynamic therapy with high-density subthreshold micropulse laser treatment in patients with chronic central serous chorioretinopathy (the PLACE trial): study protocol for a randomized controlled trial. Trials 16:419. https://doi.org/10.1186/ s13063-015-0939-Z

15. Chung CY, Chan YY, Li KKW (2018) Angiographic and tomographic prognostic factors of chronic central serous chorioretinopathy treated with half-dose photodynamic therapy. Ophthalmologica 240(1):37-44. https://doi.org/10.1159/ 000484100

16. Mohabati D, van Rijssen TJ, van Dijk EH, Luyten GP, Missotten TO, Hoyng CB, Yzer S, Boon CJ (2018) Clinical characteristics and long-term visual outcome of severe phenotypes of chronic central serous chorioretinopathy. Clin Ophthalmol 12:1061-1070. https://doi.org/10.2147/OPTH.S160956

17. Inoue R, Sawa M, Tsujikawa M, Gomi F (2010) Association between the efficacy of photodynamic therapy and indocyanine green angiography findings for central serous chorioretinopathy. Am J Ophthalmol 149(3):441-446.e441-442. https://doi.org/10.1016/j. ajo.2009.10.011

18. van Rijssen TJ, Mohabati D, Dijkman G, Theelen T, de Jong EK, van Dijk EHC, Boon CJF (2018) Correlation between redefined optical coherence tomography parameters and best-corrected visual acuity in non-resolving central serous chorioretinopathy treated with half-dose photodynamic therapy. PLoS One 13(8):e0202549. https://doi.org/10.1371/journal.pone.0202549

19. Scheider A, Nasemann JE, Lund OE (1993) Fluorescein and indocyanine green angiographies of central serous choroidopathy by scanning laser ophthalmoscopy. Am J Ophthalmol 115(1):50-56

20. Yannuzzi LA, Shakin JL, Fisher YL, Altomonte MA (1984) Peripheral retinal detachments and retinal pigment epithelial atrophic tracts secondary to central serous pigment epitheliopathy. Ophthalmology 91(12):1554-1572

21. Warrow DJ, Hoang QV, Freund KB (2013) Pachychoroid pigment epitheliopathy. Retina 33(8):1659-1672. https://doi.org/10.1097/ IAE.0b013e3182953df4

22. van Rijssen TJ, van Dijk EHC, Dijkman G, Boon CJF (2018) Clinical characteristics of chronic central serous chorioretinopathy patients with insufficient response to reduced-settings photodynamic therapy. Graefes Arch Clin Exp Ophthalmol 256(8):1395-1402. https://doi.org/10.1007/s00417-018-4003-Z

23. Ozkaya A, Garip R, Alkin Z, Taskapili M (2017) The comparison of multimodal imaging findings of central serous chorioretinopathy patients in regard to the early anatomically treatment response to half-fluence photodynamic therapy: a retrospective case-control study. Int J Retina Vitreous 3(1):20. https://doi.org/10.1186/ s40942-017-0073-Z

24. Daruich A, Matet A, Dirani A, Bousquet E, Zhao M, Farman N, Jaisser F, Behar-Cohen F (2015) Central serous chorioretinopathy: recent findings and new physiopathology hypothesis. Prog Retin Eye Res 48:82-118. https://doi.org/10.1016/j.preteyeres.2015.05. 003

Publisher's note Springer Nature remains neutral with regard to jurisdictional claims in published maps and institutional affiliations. 